\title{
Kesir Dereceli Sprott-K Kaotik Sisteminin Dinamik Analizi ve FPGA Uygulaması
}

\author{
Kenan Altun ${ }^{1 *}$ \\ 1* Sivas Cumhuriyet Üniversitesi, Sivas Teknik Bilimler MYO, Elektronik ve Otomasyon Bölümü, Sivas, Türkiye, (ORCID: 0000-0001-7419-1901), \\ kaltun@,cumhuriyet.edu.tr
}

(İlk Geliş Tarihi 19 Nisan 2021 ve Kabul Tarihi 27 Haziran 2021)

(DOI: $10.31590 /$ ejosat.922601)

ATIF/REFERENCE: Altun K. (2021). Kesir Dereceli Sprott-K Kaotik Sisteminin Dinamik Analizi ve FPGA Uygulamas1. Avrupa Bilim ve Teknoloji Dergisi, (25), 392-399.

\section{Öz}

Bu makalede, Alanda Programlanabilir Kapı Dizileri (Field Programmable Gate Array, FPGA) donanımı kullanılarak Sprott-K kaotik sisteminin kesir dereceli analiz ve deneysel uygulaması sunulmaktadır. Çalışmada, Sprott-K kaotik sisteminin ilk olarak Simulink modeli ile elde edilen çeker yapıları gerçekleştirilmiştir. Sprott-K dinamik denklemlerin matematiksel analizleri yapılarak dinamik sistemin kaosa girdiği minimum kesir derecesi belirlenmiştir. Sprott-K kaotik sisteminin tam dereceli kaotik davranışı minimum kesir dereceli sistem ile Simulink benzetimi karşılaştırılmıştır. Sistemin kesir dereceli analizi rasyonel yaklaşım modellerinden Carlson metodu kullanılarak gerçekleştirilmiştir. Carlson metodu ile sistemin kaosa girdiği kesir derecesi için frekans domenindeki transfer fonksiyonları elde edilmiştir. Elde edilen frekans domenindeki kesir dereceli transfer fonksiyonları ayrık zaman z transfer fonksiyonuna çevrilmiştir. Sistemin FPGA tasarımı, dinamik yapı Simulink kullanılarak tasarlanmıș ve MATLAB'ın HDL kod derleyicisi kullanılarak kod dönüşümü gerçekleştirilmiştir. Kaotik sistem, derleyiciden elde edilen bit akışı dosyası Xilinx FPGA ZedBoard Zynq-7000 yongasına indirilerek gerçekleştirilmiştir. Sonuçlar, FPGA yapılarının kesir dereceli kaotik sistemler için istenen doğruluk ve yüksek hızlı gerçekleştirmeler sağladığını göstermektedir.

Anahtar Kelimeler: Kaos, kesir-derecesi, Carlson metodu, Sprott-K, FPGA, HDL kodlayıc1.

\section{Dynamic Analysis of The Fractional-Order Sprott-K Chaotic System and FPGA Implementation}

\begin{abstract}
In this article, the fractional-order analysis and experimental application of the Sprott-K chaotic system using Field Programmable Gate Array (FPGA) hardware is presented. In the study, the attractor structures of the Sprott-K chaotic system, which were first obtained with the Simulink model, were realized. Mathematical analysis of Sprott-K dynamic equations was made and the minimum fractional-order at which the dynamic system entered chaos was determined. The integer-order chaotic behavior of the Sprott-K chaotic system is compared with the Simulink simulation of the minimum fractional-order system. Fractional-order analysis of the system was carried out using Carlson method, one of the rational approximation models. Transfer functions in the frequency domain are obtained for the fractional-order in which the system goes into chaos with the Carlson method. Fractional-order transfer functions in the frequency domain obtained have been converted to the discrete time $\mathrm{z}$ transfer function. The FPGA design of the system was designed using the dynamic structure Simulink and the code conversion was performed using MATLAB's HDL code compiler. Chaotic system was realized by downloading the bitstream file obtained from the compiler to the Xilinx FPGA ZedBoard Zynq-7000 chip. The results show that FPGA structures provide the desired accuracy and high speed realizations for fractional-order chaotic systems.
\end{abstract}

Keywords: Chaos, fractional-order, Carlson method, Sprott-K, FPGA, HDL coder.

\footnotetext{
*Sorumlu Yazar: kaltun@cumhuriyet.edu.tr
} 


\section{Giriş}

Kaos, kendi içinde belli bir düzen ile hareket etmesine rağmen doğrusal olmayan davranış sergileyen ve dinamik denklemlerle tanımlanan bir bilim dalıdır. Kaos tabanlı osilatör devrelerinin başlangıç değeri hassasiyeti, periyodik olmayan davranışı, tahmin edilmesi zor ve geniş bant aralığına sahip yapısı en önemli özelliklerindendir (Holmes, 1990). Sistem parametrelerindeki küçük bir değişiklik, kaotik sistem davranışında büyük bir farklılığa yol açar. Kaotik sistemler ilk olarak Fransız matematikçi Henri Poincare tarafindan tanıtılmıştır. Son zamanlarda tıp, mühendislik ve matematik gibi birçok bilimsel ve endüstriyel kullanım alanlarına sahiptir. Kaos biliminin ilk uygulaması 1960 yılında Edward Lorenz'in hava tahmini probleminin çözümünde kullanılmıştır (Lorenz, 1963). $\mathrm{Bu}$ uygulamalar, sinyal üreteçleri (Alligood ve ark., 1997), rastgele sayı üreteci (Howard, 2004), güvenilir haberleşme (Alvarez ve ark., 2006) ve kontrol sistemlerinde de karşımıza çıkmaktadır (Vaidyanathan, 2016). Özellikle IOT (internet of things) ile ilgili uygulamalarda, karmaşık yapıya sahip kaotik sistemlerin kullanılması bilgi güvenilirliğinin artırılmasına katkı sağlamıştır. Kaotik osilatörlerin ürettikleri sinyaller, gürültü benzeri ve periyodik olmayan davranışlar göstermesi, başlangıç koşullarına ve sistem parametrelerine çok hassas bir şekilde bağlı olması gibi karakteristik özelliklerinden dolayı birçok uygulamada kullanılmaktadır. $\mathrm{Bu}$ nedenle literatürde farklı özelliklere sahip birçok kaotik sistem önerilmektedir. Bu kaotik sistemlerden, sistem dinamikleri en iyi bilinenler Lorenz (Lorenz, 1963), Chua (Chua, 1992), Rössler (Rössler, 1976), Duffing sistemleri (Holmes, 1979) ve Chen (Chen ve ark., 1999) osilatörleridir.

Haberleşme sistemlerinde taşıyıcı sinyal olarak kullanılan kaos tabanlı işaretlerin tahmin edilebilirliğinin az olması bilgi sinyalinin elde edilebilmesini zorlaştıracağından haberleşme sisteminin güvenilirliğini artırmaktadır. Ayrıca kaos sinyallerinin yayılı spektruma sahip olması, bilgi sinyalinin iletim kanalı boyunca geniş band aralığında taşınacağından güvenli bir şekilde iletilmesini sağlamaktadır. Dolayısıyla kaos sinyallerinin karmaşık yapısının artması aynı zamanda kullanılan haberleşme sisteminin de yapısını karmaşık hale getirerek bilgi taşıma güvenliğini artırmaktadır. $\mathrm{Bu}$ nedenle kaotik tabanlı dinamik denklemlerin analizi ve kaos şartlarının araştırılması önem arz etmektedir. Bir sistemin kaotik davranış sergileyebilmesi için denklem takımında en az üç adet durum değişkeni ve bir adet doğrusal olmayan terim içermelidir (Peitgen ve ark., 2006). Kesir dereceli hesaplama yöntemlerinin doğrusal olmayan dinamik denklemlerin analizinde kullanılmasıyla, doğrusal olmayan bir dinamik sistemin kaotik davranış göstermesi sistem derecesinin üçten küçük olması durumunda da sağlandığı ortaya konmuştur (Petras, 2011). Böylelikle kaotik parametrelere kesir derecesinin de eklenmesiyle kaotik sistemlerin karmaşıklığı artırılmıştır (Petras, 2011).

Kesir dereceli hesaplamalar ilk olarak Leibniz tarafindan 1695 yılında incelenmesine rağmen, ilk çalışmalar Liouville ve Riemann tarafindan 19. yüzyılın sonlarında ortaya konulmuştur (Oldham ve ark., 1974). Kesir dereceli analiz tanıtılmadan önce, sistem modellemesi tamsayı dereceli analiz ile sınırlıdı. Kesir dereceli analiz ile birlikte birçok problemin çözümüne yönelik ve sistemi daha iyi tanımlayabilen analizler yapılmıştır (Oustaloup ve ark., 2000). Daha öncesinde bu modellemeler tam dereceler yakınsatılarak gerçekleştirilmiştir. Bu yaklaşımın sonucu olarak; e-ISSN: 2148-2683 ya düşük mertebeden kesin olmayan modellere ya da aşırı derecede karmaşık yüksek mertebeden modellere neden olmuştur. Kesir dereceli operatörlerin kullanılması, güncel birçok problemlerin geleneksel tamsayı dereceli modellere kıyasla daha kesin bir şekilde modellenmesine izin vermiştir (Petras, 2011). Mühendislik ve fizik alanlarındaki kesir dereceli analiz uygulamaları, sistem kontrol analizi (Caponetto ve ark., 2014), osilatör tasarımı (Rajagopal ve ark., 2018), analog filtreler (Huang ve ark., 2017) ve kaotik sistemler gibi birçok alanda artan ilgi görmektedir.

Başlangıç değeri ve parametre hassasiyeti olan kaotik tabanlı dinamik sistemlerin tam dereceli hesaplama yöntemleriyle temsil edilebilmesi mümkün değildir. Bu nedenle doğrusal olmayan dinamik sistemlerin kesir dereceli analiz yöntemlerinin kullanılmasıyla özellikle kontrol sistemleri ve elektriksel sistemlerin modellenmesi için yapılan çalışmalar artmıştır (Petras ve ark., 2011). Kesir dereceli analiz ile birlikte doğadaki birçok problemin analizi ve modellenmesiyle elde edilen çözüm yöntemlerinin daha kesin sonuçlar verdiği gözlemlenmiştir (Petras ve ark., 2011). Kesir dereceli sistemlerin ayrik ve pasif devre elemanları kullanılarak gerçekleştirilebilmesi ise oldukça zordur. Alan Programlanabilir Kapı Dizisi (FPGA) teknolojisi, karmaşık sistemlerin tasarımı ve gerçekleştirilebilmesi için önemli çözümler sunmuştur. Özellikle, kesir dereceli sistemlerde türev ve integratörlerin donanımsal gerçekleştiriminde, sistem kalitesinin artırılmasında, donanım maliyetinin azaltılması ve yüksek frekanslarda çalışabilmesine önemli katkı sağlamıştır (Jiang ve ark., 2007).

FPGA yapıları, uygulamaya özel tümleşik devreler (Application Specific Integrated Circuits (ASIC)) için prototip hazırlanmasında kullanılır (Zhang ve ark., 2008). ASIC yapılarında tasarım aşamasında aylar süren uygulama, test, hata ayıklama işlemleri FPGA yapılarında saatler içinde gerçekleştirilebilir. $\mathrm{Bu}$ nedenle tasarımda değişiklik yapmak ve sonuçları değerlendirmek kolaydır. Ayrıca, düşük güç tüketimi, düşük maliyet, kolay modifikasyon, gerçek zamanlı hesaplama ve yüksek kapasite FPGA'lerin önemli üstünlükleridir. Ayrıca FPGA yapıları yüksek frekanslarda anahtarlama hızının yanısıra, paralel işlem yapma kabiliyetine sahiptir. Mikroişlemci ve DSP (Digital Signal Processor) yapılardaki sıralı işlem hesaplaması, en az üç adet dinamik denklem içeren kaotik üreteçlerin düşük frekanslarda çalışmasına neden olur. FPGA yapılar, kaotik sistemlerin yüksek frekanslarda çalışmasına ve paralel işlem kabiliyeti ile hesaplamaların daha kısa sürede gerçekleştirilmesine imkan sağlar.

Kesir dereceli sistemlerin simülasyon ve deneysel gerçekleştiriminde pasif ve aktif devre elemanlarının kullanılmasındaki zorluklar (Jiang ve ark., 2013), esnek yapıları ve yüksek doğrulukları nedeniyle FPGA yapılarını alternatif bir seçenek haline getirmiştir (Tolba ve ark., 2017). Çalışmada kesir dereceli Sprott-K kaotik osilatörünün nümerik analizi ve FPGA tabanlı deneysel çalışması Xilinx FPGA ZYNq-7000 platformu kullanılarak gerçekleştirilmiştir. Bahsedilen çalışmada, kesir dereceli Sprott-K dinamik sisteminin analizinde Carlson metodu kullanılmıştır.

Çalışmanın ikinci bölümünde bir kesir dereceli analiz yöntemi olan Carlson metodu açıklanmış ve Sprott-K osilatörünün kesir dereceli analizi gerçekleştirilmiştir. Üçüncü bölümde Sprott-K kaotik sisteminin farklı kesir dereceleri için Carlson metodu kullanarak bilgisayar benzetimi ve kesir dereceli 
Sprott-K kaotik osilatörünün FPGA uygulaması gerçekleştirilerek elde edilen sonuçlar verilmiştir. Son bölümde ise benzetim ve deneysel çalışma sonuçları doğrulanmış, gelecek çalışmalar hakkında tartışmalara yer verilmiştir.

\section{Kesir Dereceli Analiz}

Kesir dereceli analiz, birçok sistem denklemlerinde yer alan türev alıcı ve integratörlerin genel tanımlarının yapılmasını sağlayan matematik alanında yer alan bilim dalıdır (Miller ve ark., 1993). Kesir dereceli analiz, başta kontrol sistemleri olmak üzere birçok sistemin gerçeğe en yakın modellemesinin yapılmasında önemli katkılar vermiştir (Caponetto ve ark., 2014). Analiz yöntemi ilk olarak, Leibniz ve L'Hopital, tarafından 0,5 dereceli türev alıcının araştırılmasıyla başlamıştır (Ross, 1977). Kesir dereceli analizlerde; hesaplama, karmaşık ve zahmetli süreçleri de beraberinde getirmektedir. Özellikle kontrol sistemlerinin analiz ve simülasyonlarını kolaylaştırmak amacıyla, kesir dereceli operatörlerin tam dereceli transfer fonksiyonlarına yakınsatılması düşünülmüştür. Bunun için literatürde sıkça karşılaşılan ve kullanılan birçok yöntem önerilmiştir (Charef ve ark., 1992).

Kesir dereceli türev hesaplamasında, kesir dereceli türev operatörü komşu tamsayı dereceli türevleri arasında bir enterpolasyon olarak davranır. Kesir dereceli hesaplanan sistemlerde kesir derecesi ekstra bir parametre olarak, tüm sistemin davranışını gerçeğe en yakın modellemek için kullanılır. Kesir dereceli sistemlerin bu özelliği, doğrusal olmayan sistemlerin modellenmesine önemli katkı sağlamaktadır.

Kesir dereceli sistemlerin analizi için birçok yaklaşım metodu önerilmiştir: Carlson metodu, Matsuda metodu, Oustaloup metodu, Grünwald-Letnikoff yaklaşım metodu, vb. (Podlubny, 1999). Gerçekleştirilen bu çalışmada Carlson yaklaşım metodu kullanılmıştır.

\subsection{Carlson Metodunun Teorik Analizi}

$\mathrm{Bu}$ method, Carlson tarafindan (Carlson ve ark, 1964) önerilen Newton kanunundan elde edilen ve $\alpha$ kökün yinelemeli yaklaşımı olarak kabul edilebilir. Yöntemin başlangıç noktası, Newton teorisindeki devamlı kesir genişlemesi ilkesine dayanmaktadır. Yöntemde kesir dereceli operatör denklem (1) ile aşağıdaki gibi tanımlanmıştır.

$$
F(s)=s^{\alpha}, \quad \alpha \in R .
$$

Denklem (2) ile verilen ifadede Newton'un yinelemeli süreci kullanılarak, yaklaşık rasyonel fonksiyon $F_{i}(s)$ elde edilir. Burada; $s=j \omega$ için kompleks frekansı ve $\alpha$ pozitif kesirli integratör derecesini $i$ kesir derecesinin mertebesini temsil etmektedir.

$$
F_{i}(s)=F_{i-1}(s) \frac{\left(\frac{1}{\alpha}-1\right) F_{i-1}^{\frac{1}{\alpha}}(s)+\left(\frac{1}{\alpha}+1\right) s}{\left(\frac{1}{\alpha}+1\right) F_{i-1}^{\frac{1}{\alpha}}(s)+\left(\frac{1}{\alpha}-1\right) s} \quad, \quad i \in N
$$

$\mathrm{F}_{0}(\mathrm{~s})=1$ olarak başlangıç değeri ile tanımlandığında, 1/a 'nın sadece $\pm 2, \pm 3 \ldots . \pm \mathrm{n}, \forall \mathrm{n} \in N$ tamsayı değerleri ile hesaplanması gerekir. Buna göre, sadece $\mathrm{S}^{ \pm 0,1}, \mathrm{~S}^{ \pm 0,2}$ değerleri hesaplandıktan sonra, $S^{ \pm 0,5}$ ve $S^{ \pm 1 / 3}, S^{ \pm 1,4}$ v.b. değerler bu yöntemle doğrudan elde edilebilir (Oliveira Valério, 2005).

\subsection{Sprott-K Osilatörünün Kesir Derecesi Analizi}

e-ISSN: 2148-2683
Kesir dereceli analizi elde edilecek kaotik osilatörlerden Sprott-K kaotik üretece ait dinamik sistemi Denklem (3) ile verilmiştir (Sprott, 1994). Kaotik sisteme ait dinamik denklemler üç boyut ve yedi terimden oluşmaktadır.

$$
\begin{aligned}
& D_{t}^{q 1} x(t)=x(t) \cdot y(t)-z(t) \\
& D_{t}^{q 2} y(t)=x(t)-y(t) \\
& D_{t}^{q 3} z(t)=x(t)+0.3 \cdot z(t),
\end{aligned}
$$

burada $\mathrm{q}_{1}, \mathrm{q}_{2}$ ve $\mathrm{q}_{3}$ kesirli türev alıcının dereceleridir.

Transfer fonksiyonu elde edilecek sistemde, seçilecek minimum kesir derecesi için dinamik sistemin öz değerleri hesaplanır. Hesaplanan bu öz değerlerin aşağıdaki teorem kullanılarak her bir öz değer için bir kesir derecesi hesaplanır. Sistemin kesir derecesi ise bu değerlerin en büyüğü olarak seçilir (Matignon, 1996).

Buna göre;

Öncelikle $\mathrm{n}$ boyutlu bir dinamik sistemde her bir boyut için Jacobian matrisleri elde edilerek öz değerleri denklem (4) ile verilen ifade kullanılarak $\left(\lambda_{1}, \lambda_{2}, . ., \lambda_{\mathrm{n}}\right)$ bulunur. Elde edilen her bir öz değer için denklem (5) ile verilen ifadede $\alpha$ kesir derecesi belirlenir. Sistemin kararlılığı kök dereceleri hesaplanarak belirlenir.

$$
\begin{gathered}
\mid \arg \left(\lambda_{i} \mid>\alpha \pi / 2,\right. \\
\alpha=\max \left(q_{1}, q_{2}, \ldots, q_{n}\right)(i=1,2, \ldots, n) .
\end{gathered}
$$

Kaotik tabanlı bir sistem davranışı periyodik davranış sergiliyorsa sistem kararlıdır. Sistemin kararlı olduğu kesir dereceli aralıkta kaotik sistemde kararlı davranış sergileyerek periyodik olacaktır. Sistemin kararsız olduğu ve denklem (4) ile hesaplanan aralıkta ise sistem kaotik davranış sergileyecektir. Kesir dereceli sistemlerin kararlılık teorisi Şekil 1 ile gösterilmiştir. Buna göre kaotik sistemin kararsız durumları daha hassas belirlenerek kaosa girdiği dereceler kesirli olarak ifade edilebilir. Böylelikle kesir derecelerinde de kaotik davranış sergileyen dinamik sistemler elde edilmiş olur.

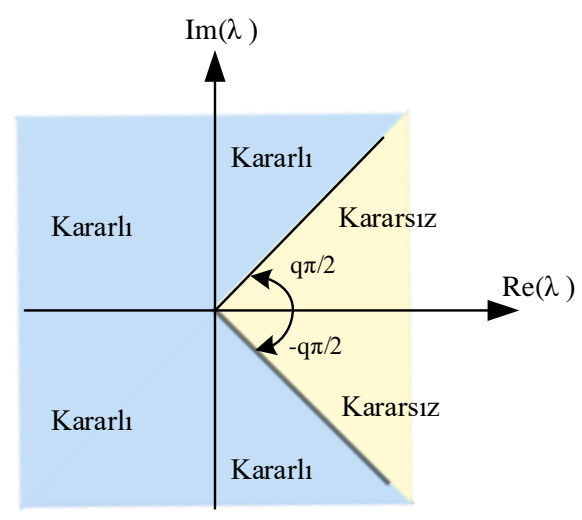

\section{Şekil 1. Kesir dereceli sistemlerde kararlı bölgeler.}

Analizi yapılacak kesir dereceli bir sistemin Jacobian matrisinin öz değerlerinin $\lambda_{1}, \lambda_{2}$ ve $\lambda_{3}$ olduğunu varsayalım. $\mathrm{Bu}$ durumda her bir öz değer için kesir derecesi hesaplanır. $\arg \left(\lambda_{1}\right)$, $\arg \left(\lambda_{2}\right)$ ve $\arg \left(\lambda_{3}\right)$ elde edilir. Bu durumda sistemin kesir derecesi $\max \left(\mathrm{q}_{1}, \mathrm{q}_{2}, \mathrm{q}_{3}\right)$ ifadesinden $\alpha=\mathrm{q}_{1}=\mathrm{q}_{2}=\mathrm{q}_{3}$ eşit olur. $\mathrm{q}_{1}, \mathrm{q}_{2}$ ve $\mathrm{q}_{3}$ 'ün 
hepsi $\alpha$ 'dan büyük olduğunda, tüm denge noktaları kararsızdır ve dinamik sistemin kaosa girmesi mümkündür.

Kesir dereceli analizi yapılacak Sprott-K kaotik sisteminde başlangıç şartları $\mathrm{x}_{0}=0.5, \mathrm{y}_{0}=0.5, \mathrm{z}_{0}=0.5$ olarak alınarak Jacobian matrisinin öz değerleri $\lambda_{1}=-1, \lambda_{2}=0.15+\mathrm{i} 0.988686$ ve $\lambda_{3}=0.15$ - i0.988686 olarak bulunmuştur. Bu durumda her bir öz değer için hesaplanan kesir dereceleri denklem (4)'deki gibi arg $\left(\lambda_{1}\right)=\pi$, arg $\left(\lambda_{2}\right)=1.4203$ ve $\arg \left(\lambda_{3}\right)=-1.4203$ olarak bulunmuştur. $\mathrm{Bu}$ durumda kesir derecesi $\max \left(\mathrm{q}_{1}, \mathrm{q}_{2}, \mathrm{q}_{3}\right)$ ifadesinden $\alpha=\mathrm{q}_{2}=\mathrm{q}_{3}=0,9041$ eşit olur. Çalışmada dinamik denklemlerin integratör dereceleri birbirine eşit olarak 0,89-0,93 aralığında seçilip bir sonraki bölümde simülasyon ve deneysel uygulamada kullanılmıştır.

\section{Kesir Dereceli Sprott-K Osilatörü}

Sprott-K kaotik osilatörünün analizi neticesinde elde edilen minimum kesir derecesi $\alpha=0,91$ alınarak Carlson yaklaşım metodu ile s domeninde transfer fonksiyonu elde edilmiştir. Denklem (7)'de hesaplanan birinci dereceden transfer fonksiyonu için denklem (6) kullanılmış ve $i=1, F_{0}(s)=1$ alınarak elde edilmiştir.

$$
\begin{array}{r}
F_{i}(s)=F_{i-1}(s) \frac{\left(\frac{1}{\alpha}-1\right) F_{i-1}^{\frac{1}{\alpha}}(s)+\left(\frac{1}{\alpha}+1\right) s}{\left(\frac{1}{\alpha}+1\right) F_{i-1}^{\frac{1}{\alpha}}(s)+\left(\frac{1}{\alpha}-1\right) s}, \\
F_{1}(s)=\frac{9 s+191}{191 s+9} .
\end{array}
$$

Elde edilen kesir dereceli transfer fonksiyonu Sprott-K kaotik sistemin Simulink tasarımında kullanılmıştır.

\subsection{Sprott-K Sisteminin Bilgisayar Benzetimi}

Bir önceki bölümde kesir derecesi ve transfer fonksiyonu belirlenen Sprott-K kaotik sisteminin kaosa girmeden ve girdikten sonra elde edilen kaotik davranışları, faz-uzay gösterimleri ile ortaya konmuştur. Sistemin Simulink modellemesinde tasarım kolaylığı sağlayacağından ilk olarak Laplace domenindeki transfer fonksiyonu kullanılmıştır. Simulink kullanılarak tasarlanan kesir dereceli Sprott-K kaotik sistemine ait blok şema Şekil 2'de verilmiştir.

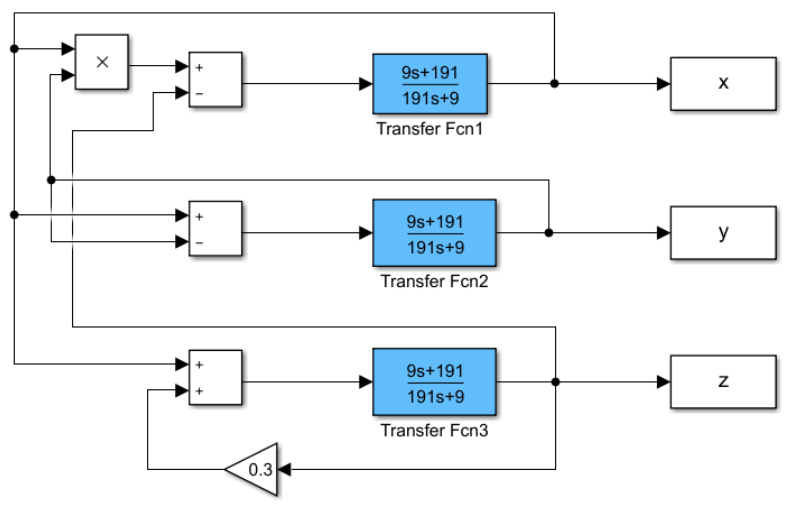

Şekil 2. Kesir dereceli Sprott-K kaotik sistemin Simulink blok
seması.

Şekil 3 incelendiğinde Sprott-K sisteminin $\mathrm{q}=0,89$ kesir derecesi için kararlı bir sistem davranışı sergilediği görülmektedir. Her üç faz-uzay gösteriminde de kaos davranış sergilemeyen dinamik sistem çıkışları elde edilmiştir.

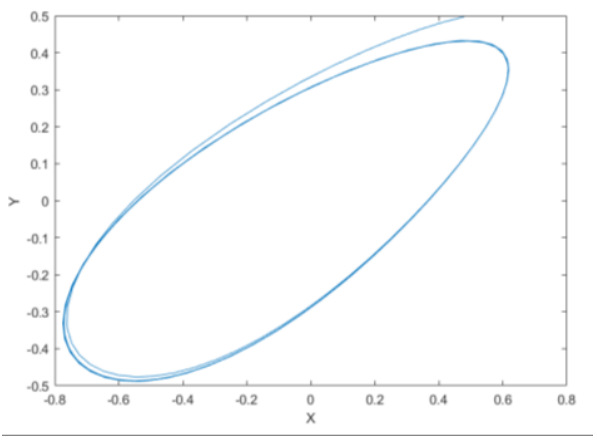

(a)

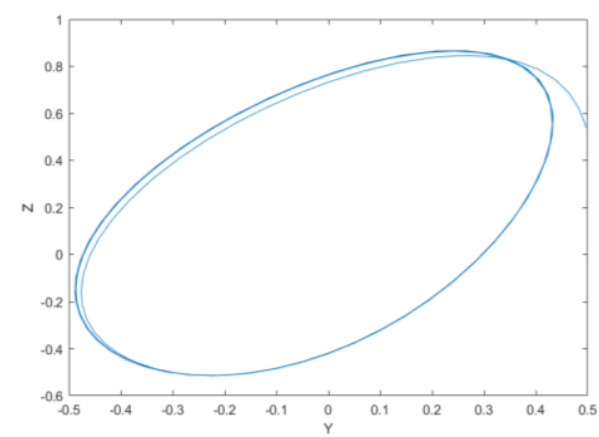

(b)

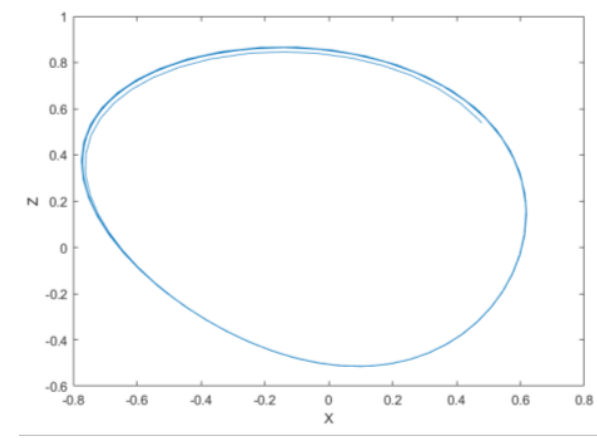

(c)

Şekil 3. Sprott-K kaotik sisteminin $q=0,89$ kesir derecesi için elde edilen a) $x-y, b$ ) $y-z$, c) $x-z$ faz-uzay gösterimleri. 
Şekil 4 incelendiğinde Sprott-K sisteminin $\mathrm{q}=0,91 \mathrm{kesir}$ derecesinde kararsız yapıya geçmeye başladığı ve kararsız bir sistem davranış1 sergilediği görülmektedir. Her üç faz-uzay gösteriminde de 3 . ve 4. pencerelerin açıldığı gözlemlenmekte ve sistem kaos davranış sergilemeye başlamaktadır.

Sprott-K kaotik sistem için yapılan kaos derecesi analizinde kararsız yapının $q=0,9041$ kesir derecesinden büyük değerlerde gerçekleştirildiği belirlenmişti. Yapılan simülasyon çalışması ile bu değer doğrulanmıştır.

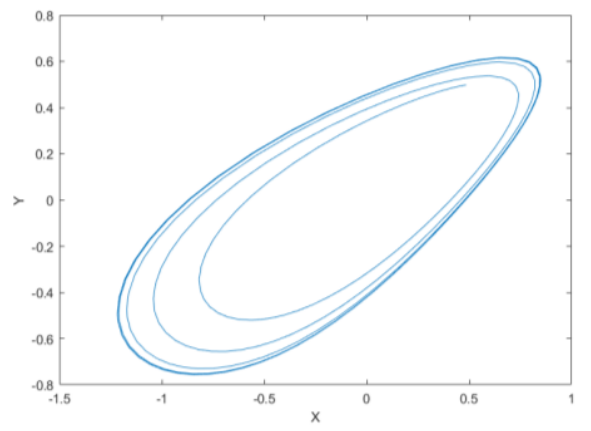

(a)

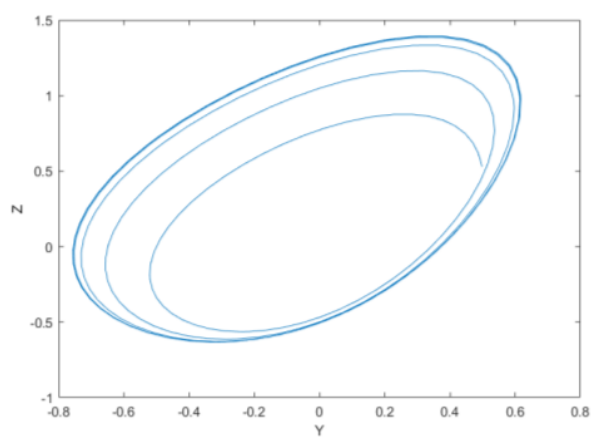

(b)

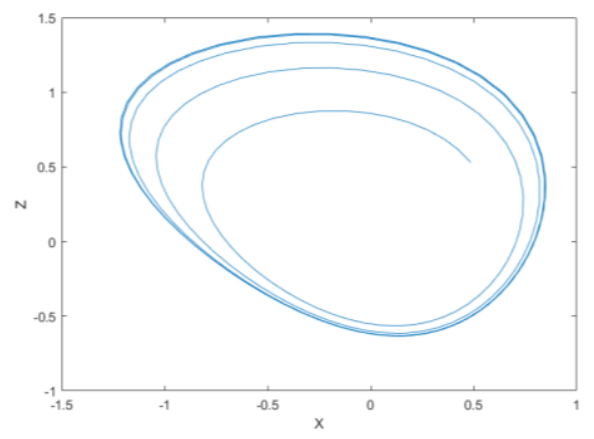

(c)

Şekil 4. Sprott-K kaotik sisteminin $q=0,91$ kesir derecesi için elde edilen a) $x-y, b) y-z$, c) $x-z$ faz-uzay gösterimleri.

Ayrıca kesir derecesi $\mathrm{q}=0,93$ seçilerek çalışma tekrarlandığında Şekil 5'deki faz-uzay gösterimleri elde edilmiştir. Sistemin kararsız davranışı devam etmekte ve kaotik davranış sergilemektedir.

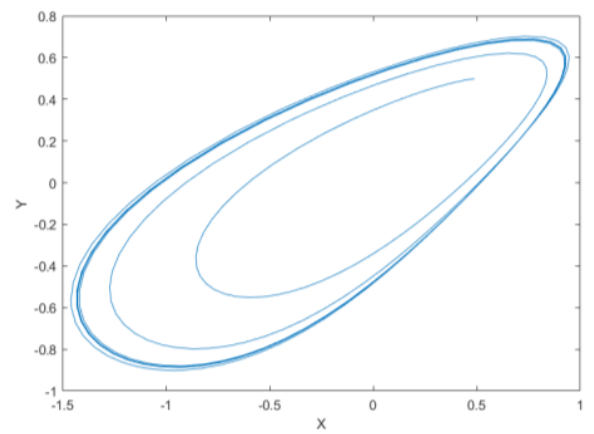

(a)

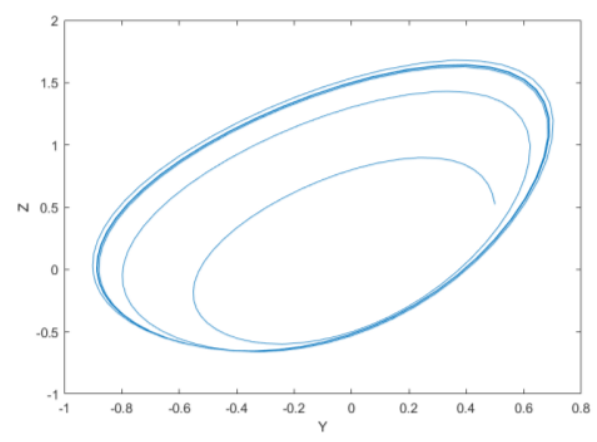

(b)

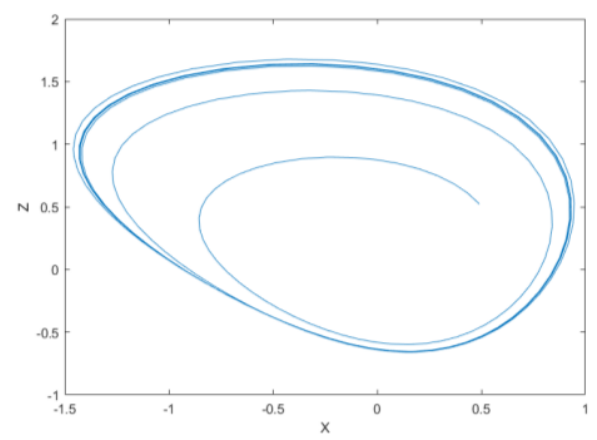

(c)

Şekil 5. Sprott-K kaotik sisteminin $q=0,93$ kesir derecesi için elde edilen a) $x-y, b) y-z$, c) $x$-z faz-uzay gösterimleri.

Simülasyon sonuçlarına göre minimum kesir derecesi $\mathrm{q}=$ 0,9041 değerinin altında sistem periyodik davranış sergilemiştir. Kesir derecesi $\mathrm{q}=0,91$ ve $\mathrm{q}=0,93$ için tekrarlanan simülasyonda sistem kaotik davranış sergilemeye başlamıştır.

\subsection{Sprott-K Sisteminin FPGA Gerçekleştirimi}

FPGA tabanlı kesir dereceli kaotik sistemlerin birçok uygulaması literatürde gerçekleştirilmiştir (Shah ve ark., 2017). $\mathrm{Bu}$ kesir dereceli kaotik sistemler Grünwald-Letnikov metodu kullanılarak MATLAB'ın HDL kodlayıcısı ile FPGA yapılarında gerçekleştirilmiştir (Tolba ve ark., 2017). HDL kodlayıcı Simulink'te Xilinx (Vivado) sistem blokları kullanılarak elde edilmiştir (Günay ve ark., 2018).

Bu çalışmanın amacı, Sprott-K kaotik sisteminin kesir dereceli analiz, simülasyon ve deneysel uygulamasını kapsamaktadır. Sistem parametreleri ve uygun kesir derecesi için kaotik sistem tamsayı dereceli sistem ile benzer karakteristik davranış sergilemektedir. Makale, Carlson metodu kullanılarak elde edilen transfer fonksiyonunun doğrulanmasını 
araştırmaktadır. Kaotik sistem, Şekil 6'da verilen blok şemada da gösterildiği gibi ilk önce Simulink tasarımı ile Xilinx system Generator kullanılarak elde edilen VHDL dili Xilinx ISE üzerinden simüle edilmiş ve ardından ZedBoard Zynq-7000 FPGA üzerinde gerçekleştirilmiştir.

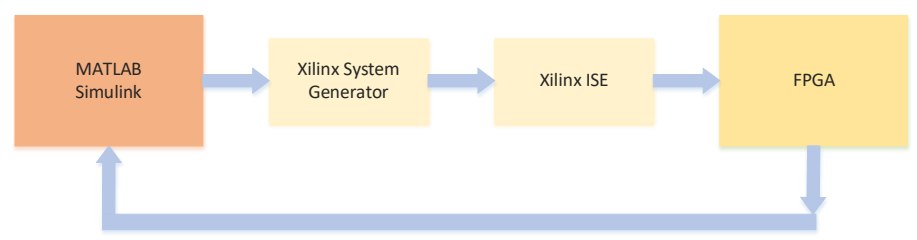

Şekil 6. FPGA tabanlı sistemlerde simülasyon blok şeması.

Sprott-K kaotik osilatörünün FPGA tasarımı için ilk olarak s domeninde belirlenen kesir derecesi için elde edilen denklem (8) ile verilen transfer fonksiyonu ayrık zaman domeninde tekrar hesaplanmıştır. Sistemin z transformu, Matlab'da “ $c 2 d$ ” komutu kullanılarak aşağıdaki gibi elde edilmiştir:

$$
\begin{gathered}
F_{1}(s)=\frac{9 s+191 s}{191 s+9}, \\
\text { 'sysd }=c 2 d\left(s y s c, T_{s}\right) ' \\
\text { 'sysd }=c 2 d(\text { sysc, } 0,01)^{\prime}, \\
F_{1}(z)=\frac{0,047112-0,03712}{z-0,9995} .
\end{gathered}
$$

Burada denklem (9)'da verilen $\mathrm{z}$ domenindeki transfer fonksiyonu ile Şekil 7'deki Sprott-K kaotik sistemin ayrik zaman Simulink tasarımı gerçekleştirilmiştir. Yapılan çalışmanın deneysel uygulama devresi ise Şekil 8 ile verilmektedir. Deneysel çalışma sonuçları dijital osiloskop kullanılarak elde edilmiş ve Şekil 9 ile $\mathrm{q}=0,91$ kesir derecesi için $\mathrm{x}-\mathrm{y}$, y-z ve $\mathrm{x}-\mathrm{z}$ faz uzay gösterimleri verilmektedir.

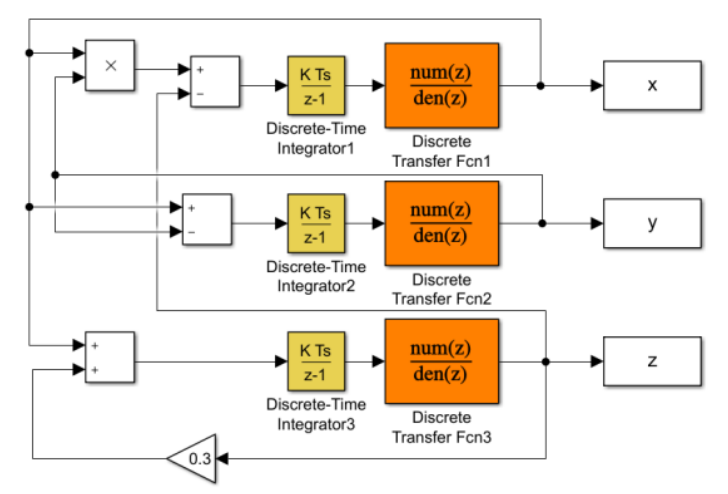

Şekil 7. Kesir dereceli Sprott-K kaotik sistemin ayrık zaman Simulink blok şemasi.

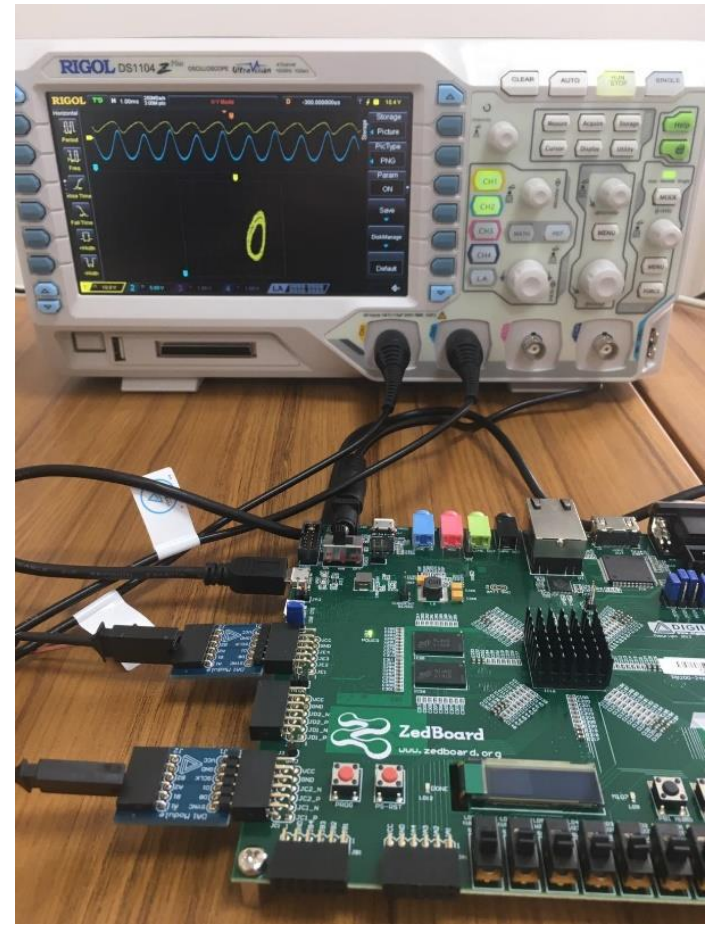

Şekil 8. Kesir dereceli kaotik sistemin FPGA uygulama devresi.

Gerçekleştirilen deneysel çalışma neticesinde elde edilen sonuçlar, simülasyon sonuçları ile benzerlik göstermektedir. Carlson metodu ile elde edilen kesir dereceli kaotik sistemin FPGA tabanlı deneysel uygulaması gerçekleştirilmiştir.

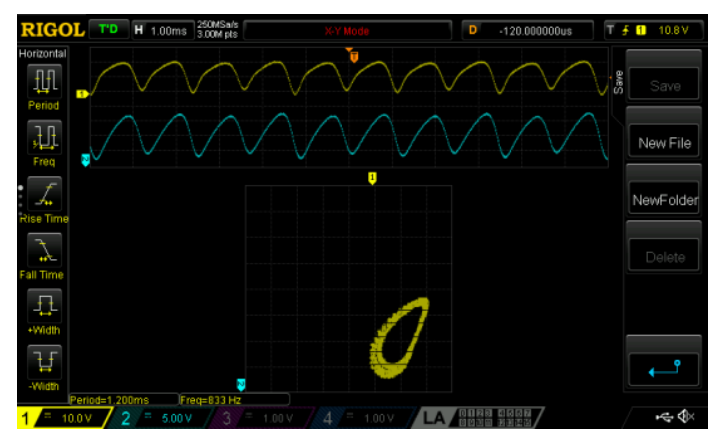

(a)

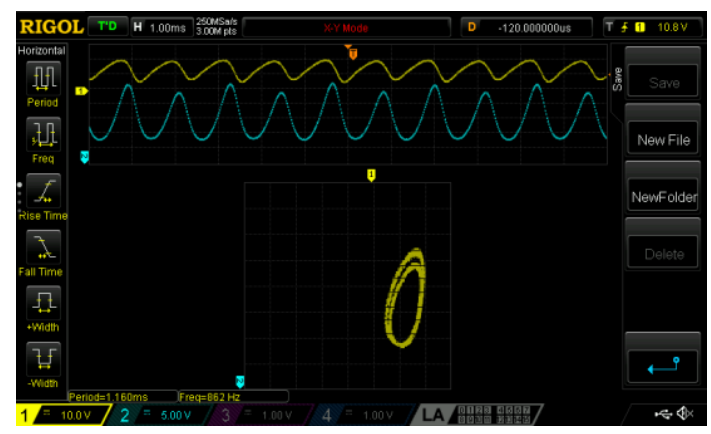

(b) 


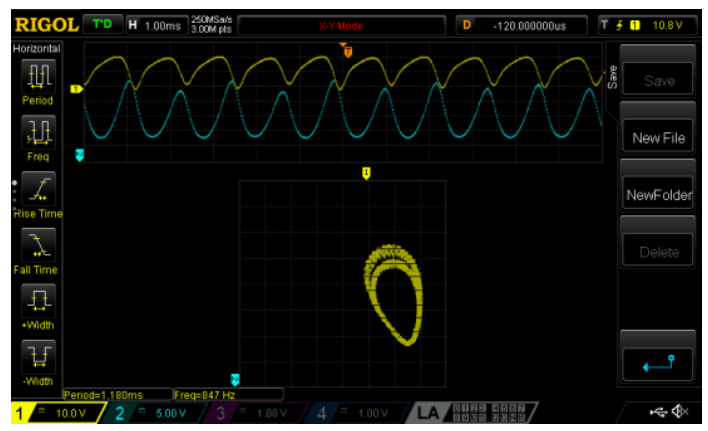

(c)

Şekil 9. Sprott-K kaotik sisteminin $q=0,91$ kesir derecesi için FPGA platformunda elde edilen a) $x-y, b$ ) $y-z$, c) $x-z$ fazuzay ve zaman eksenindeki gösterimleri.

FPGA yapıları kullanılarak gerçekleştirilen bu çalışma ile doğrusal olmayan dinamik sitemlerin yakınsatılmış tam dereceli sonuçları yerine kesir dereceli gerçek sistem davranışları elde edilmiştir. Böylelikle modellenen kaotik sistemin tam olarak davranışları gözlemlenmiştir.

\section{Sonuç}

Bu çalışmada kesir dereceli kaotik sistemlerin sayısal tabanlı uygulaması sunulmuştur. Sprott-K kaotik osilatörün Carlson metodu kullanılarak minimum kesir derecesi için transfer foksiyonu elde edilmiştir. MATLAB Simulink kullanılarak simülasyonu gerçekleştirilmiştir. Çalışmada Xilinx ZedBoard Zynq-7000 FPGA geliştirme kartı kullanılarak kesir dereceli kaotik sistem tasarım ve deneysel gerçekleştirimi yapılmıştır. Deneysel çalışmalarda elde edilen sonuçlar simülasyon sonuçları ile örtüşmektedir.

Çalışma neticesinde elde edilen sonuçlardan, kesir dereceli kaotik osilatörlerde kesir derecesi yeni bir parametre olarak haberleşme sisteminin tahmin edilebilirliğini zorlaştırmıştır. Ayrıca kesir dereceli kaotik sistemlerin hyper kaotik davranış sergilediği de gözlemlenmiştir. Bu durum haberleşme sistemleri için kanal sayısının artmasını ve bit hata oranının azalmasını sağlayacağı düşünülmektedir. Bir sonraki çalışma, elde edilen bu sonuçlar dikkate alınarak hedeflenmektedir.

\section{Teşekkür}

$\mathrm{Bu}$ çalışma, Sivas Cumhuriyet Üniversitesi Bilimsel Araştırma Projeleri (CÜBAP) tarafından SMYO-029 proje numarası ile desteklenmiştir.

\section{Kaynakça}

Alligood, K. T., Sauer, T. D., \& Yorke, J. A. (1997). Chaos in differential equations. In Chaos (pp. 359-397). Springer, Berlin, Heidelberg.

Alvarez, G., \& Li, S. (2006). Some basic cryptographic requirements for chaos-based cryptosystems. International journal of bifurcation and chaos, 16(08), 2129-2151.

Caponetto, R., Dongola, G., Maione, G., \& Pisano, A. (2014). Integrated technology fractional order proportional-integralderivative design. Journal of Vibration and Control, 20(7), 1066-1075.
Carlson, G., \& Halijak, C. (1964). Approximation of fractional capacitors $(1 / \mathrm{s})^{\wedge}(1 / \mathrm{n})$ by a regular Newton process. IEEE Transactions on Circuit Theory, 11(2), 210-213.

Charef, A., Sun, H. H., Tsao, Y. Y., \& Onaral, B. (1992). Fractal system as represented by singularity function. IEEE Transactions on automatic Control, 37(9), 1465-1470.

Chen, G., \& Ueta, T. (1999). Yet another chaotic attractor. International Journal of Bifurcation and chaos, 9(07), 1465-1466.

Chua, L. O. (1992). The genesis of Chua's circuit. Berkeley, CA, USA: Electronics Research Laboratory, College of Engineering, University of California.

Günay, E., \& Altun, K. (2018). Güvenilir Haberleşmede Açık Kapalı Kaotik Anahtarlama Sisteminin FPGA Kullanılarak Gerçekleştirilmesi. Selçuk Üniversitesi Mühendislik, Bilim ve Teknoloji Dergisi, 6(4), 559-571.

Holmes, P. (1979). A nonlinear oscillator with a strange attractor. Philosophical Transactions of the Royal Society of London. Series A, Mathematical and Physical Sciences, 292(1394), 419-448.

Holmes, P. (1990). Poincaré, celestial mechanics, dynamicalsystems theory and "chaos". Physics Reports, 193(3), 137163.

Howard, R. M. (2004). Principles of random signal analysis and low noise design: The power spectral density and its applications. John Wiley \& Sons.

Huang, X., Zhang, B., Qin, H., \& An, W. (2017). Closed-form design of variable fractional-delay FIR filters with low or middle cutoff frequencies. IEEE Transactions on Circuits and Systems I: Regular Papers, 65(2), 628-637.

Jiang, C. X., Carletta, J. E., \& Hartley, T. T. (2007). Implementation of fractional-order operators on field programmable gate arrays. In Advances in fractional calculus (pp. 333-346). Springer, Dordrecht.

Jiang, C. X., Carletta, J. E., Hartley, T. T., \& Veillette, R. J. (2013). A systematic approach for implementing fractional-order operators and systems. IEEE Journal on Emerging and Selected Topics in Circuits and Systems, 3(3), 301-312.

Lorenz, E. N. (1963). Deterministic nonperiodic flow. Journal of atmospheric sciences, 20(2), 130-141.

Matignon, D. (1996, July). Stability results for fractional differential equations with applications to control processing. In Computational engineering in systems applications (Vol. 2, No. 1, pp. 963-968).

Miller, K. S., \& Ross, B. (1993). An introduction to the fractional calculus and fractional differential equations. Wiley.

Oldham, K., \& Spanier, J. (1974). The fractional calculus theory and applications of differentiation and integration to arbitrary order. Elsevier.

Oliveira Valério, D. P. M. (2005). Fractional robust system control. Universidade Técnica de Lisboa.

Peitgen, H. O., Jürgens, H., \& Saupe, D. (2006). Chaos and fractals: new frontiers of science. Springer Science \& Business Media.

Petráš, I. (2011). Fractional-order chaotic systems. In Fractionalorder nonlinear systems (pp. 103-184). Springer, Berlin, Heidelberg.

Petráš, I. (2011). Fractional-order nonlinear systems: modeling, analysis and simulation. Springer Science \& Business Media.

Podlubny, I. (1999). An introduction to fractional derivatives, fractional differential equations, to methods of their solution and some of their applications. Mathematics in science and engineering, 198, xxiv+-340. 
Rajagopal, K., Akgul, A., Jafari, S., \& Aricioglu, B. (2018). A chaotic memcapacitor oscillator with two unstable equilibriums and its fractional form with engineering applications. Nonlinear Dynamics, 91(2), 957-974.

Ross, B. (1977). The development of fractional calculus 1695 1900. Historia Mathematica, 4(1), 75-89.

Rössler, O. E. (1976). An equation for continuous chaos. Physics Letters A, 57(5), 397-398.

Shah, D. K., Chaurasiya, R. B., Vyawahare, V. A., Pichhode, K., \& Patil, M. D. (2017). FPGA implementation of fractionalorder chaotic systems. AEU-International Journal of Electronics and Communications, 78, 245-257.

Sprott, J. C. (1994). Some simple chaotic flows. Physical review E, $50(2)$, R647.

Tolba, M. F., AbdelAty, A. M., Soliman, N. S., Said, L. A., Madian, A. H., Azar, A. T., \& Radwan, A. G. (2017). FPGA implementation of two fractional order chaotic systems. AEU-International Journal of Electronics and Communications, 78, 162-172.

Vaidyanathan, S. (2016). Generalized projective synchronization of vaidyanathan chaotic system via active and adaptive control. In Advances and Applications in Nonlinear Control Systems (pp. 97-116). Springer, Cham.

Zhang, Y., Liu, Z., \& Zheng, X. (2008, December). A chaos-based image encryption ASIC using reconfigurable logic. In APCCAS 2008-2008 IEEE Asia Pacific Conference on Circuits and Systems (pp. 1782-1785). IEEE. 